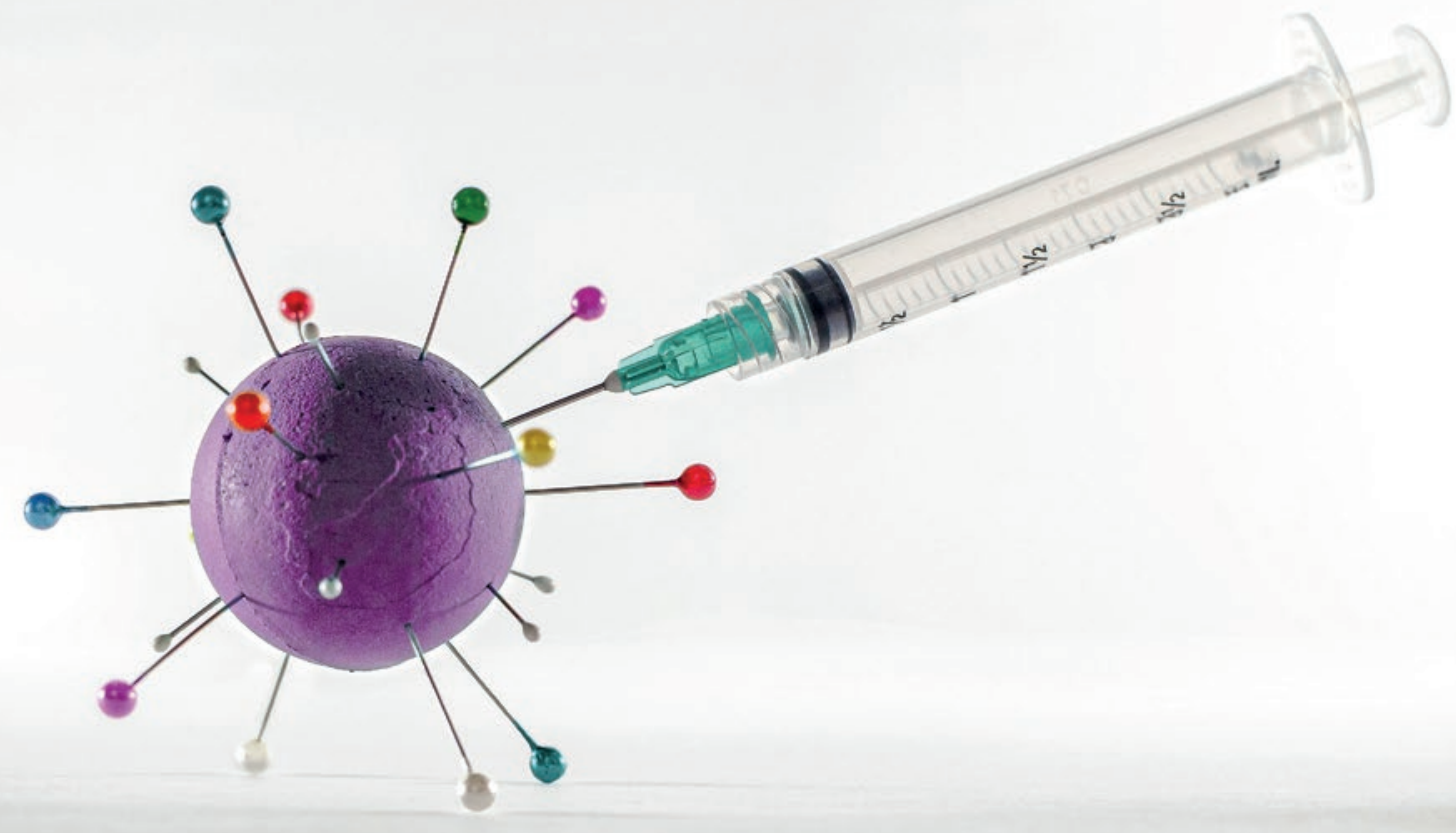

\title{
Impfobligatorium: Der rechtliche Rahmen
}

\section{Iris Herzog-Zwitter}

Dr. iur., Abteilung Rechtsdienst FMH

Ist ein Impfobligatorium rechtlich zulässig, und wenn ja, unter welchen Umständen? Diesen Fragen geht der folgende Artikel nach. Die Autorin berichtet, wie der Europäische Gerichtshof für Menschenrechte dieses Thema beurteilt, legt die Grundlagen der Schweizer Gesetzgebung dazu dar und beleuchtet das Impfobligatorium aus arbeitsrechtlicher Sicht.

Die Pandemie und das Thema «Impfobligatorium» beschäftigen nicht nur die Politik, die Medizin und die juristische Lehre, sondern zunehmend auch die Gerichte. Aktuell hat sich der Europäische Gerichtshof für Menschenrechte (EGMR) zum Impfobligatorium im Zusammenhang mit Art. 8 EMRK «Recht auf Achtung des Privat- und Familienlebens» geäussert. Am 8. April 2021 hat der Europäische Gerichtshof für Menschenrechte im Fall «VAVŘIČKA und Andere/Tschechien» mit der Mehrheit von 16:1 entschieden, dass ein Impfobligatorium zum Schutz vor gefährlichen Krankheiten menschenrechtskonform sei. Im Zentrum des vorliegenden Verfahrens stand die tschechische
Impfverordnung. Diese sieht unter anderem ein Impfobligatorium von Kindern vor. Wer gegen dieses Impfobligatorium verstösst, wird gebüsst, und ein Betreuungsplatz im Kindergarten kann in der Folge verwehrt werden [1].

Aus dem fast 100-seitigen Urteil werden nachfolgend zwei Direktzitate wiedergegeben, aus welchen grundlegende Überlegungen des EGMR hervorgehen.

"As for the effectiveness of vaccination, the Court refers once again to the general consensus over the vital importance of this means of protecting populations against diseases that may have severe effects on individual health, and that, in the case of serious 
outbreaks, may cause disruption to society [...] The Court accepts that the exclusion of the applicants from preschool meant the loss of an important opportunity for these young children to develop their personalities and to begin to acquire important social and learning skills in a formative pedagogical environment. However, that was the direct consequence of the choice made by their respective parents to decline to comply with a legal duty, the purpose of which is to protect health, in particular in that age group. Moreover, the possibility of attendance at preschool of children who cannot be vaccinated for medical reasons depends on a very high rate of vaccination among other children against contagious diseases. The Court considers that it cannot be regarded as disproportionate for a State to require those for whom vaccination represents a remote risk to health to accept this universally practised protective measure, as a matter of legal duty and in the name of social solidarity, for the sake of the small number of vulnerable children who are unable to benefit from vaccination. In the view of the Court, it was validly and legitimately open to the Czech legislature to make this choice, which is fully consistent with the rationale of protecting the health of the population. The notional availability of less intrusive means to achieve this purpose, as suggested by the applicants, does not detract from this finding."

Es ist davon auszugehen, dass das Urteil VAVŘIČKA Auswirkungen auf die aktuelle gesellschaftspolitische und juristische Auseinandersetzung zum Thema Impfobligatorium auch in der Schweiz zeigen wird. $\mathrm{Zu}$ verweisen ist auf ein Leiturteil des Bundesgerichts vom 8. Juli 2021, welches das Urteil VAVŘIČKA zitiert im Zusammenhang mit der Verhältnismässigkeit von Massnahmen, um die Verbreitung übertragbarer Krankheiten in der Bevölkerung oder in bestimmten Personengruppen zu verhindern [2].

\section{Bundesverfassung}

Ausgehend von Art. 118 BV ist der Bund verpflichtet, Vorschriften bzgl. der Bekämpfung übertragbarer, stark verbreiteter Krankheiten zu erlassen.

Der Wortlaut von Art. 118 BV ist wie folgt:

1 Der Bund trifft im Rahmen seiner Zuständigkeiten Massnahmen zum Schutz der Gesundheit.

2 Er erlässt Vorschriften über:

a. den Umgang mit Lebensmitteln sowie mit Heilmitteln, Betäubungsmitteln, Organismen, Chemikalien und Gegenständen, welche die Gesundheit gefährden können;

b. die Bekämpfung übertragbarer, stark verbreiteter oder bösartiger Krankheiten von Menschen und Tieren;

c. den Schutz vor ionisierenden Strahlen.

\section{Epidemiengesetz (EpG)}

Gestützt auf Art. 118 Abs. 2 BV erfolgte der Erlass des Bundesgesetzes über die Bekämpfung übertragbarer Krankheiten des Menschen (Epidemiengesetz). Dieses Gesetz bezweckt, den Ausbruch und die Verbreitung übertragbarer Krankheiten zu verhüten und zu bekämpfen [3]. Das EpG sieht als Grundprinzip die Arbeitsteilung von Bund und Kantonen vor. Der Bund hat die Oberaufsicht des Vollzugs des EpG und koordiniert falls nötig die kantonalen Massnahmen.

Das EpG wurde vom Bundesrat durch die Verordnung (VO) über die Bekämpfung übertragbarer Krankheiten des Menschen (Epidemienverordnung, EpV) [4] sowie die VO über mikrobiologische Laboratorien ergänzt. Dazu existiert eine EDI-VO über die meldepflichtigen Beobachtungen übertragbarer Krankheiten des Menschen.

\section{Covid-19-Gesetz}

Dieses Gesetz regelt besondere Befugnisse des Bundesrates zur Bekämpfung der Covid-19-Epidemie und zur Bewältigung der Auswirkungen der Bekämpfungsmassnahmen auf Gesellschaft, Wirtschaft und Behörden.

«Die Gesetzesvorlage fasst Massnahmen aus zahlreichen Sachgebieten zusammen. Zwischen diesen Massnahmen besteht ein enger sachlicher Zusammenhang, womit der Grundsatz der Einheit der Materie respektiert wird: Die Vorlage ist als homogenes Massnahmenpaket konzipiert, das alle zur Bewältigung der Covid-19-Epidemie bereits ergriffenen und noch erforderlichen Regelungen zusammenfasst. Diese Primär- und Sekundärmassnahmen dienen dem gleichen Zweck und regeln in vergleichbarer Weise, wie der Bundesrat zur Bewältigung der Epidemie und ihrer Auswirkungen für eine befristete Zeit von den regulären gesetzlichen Bestimmungen abweichen darf» [5].

\section{Kantonale Kompetenz}

Die Kompetenz der Kantone ist gemäss Art. 22 «Obligatorische Impfungen» EpG insofern gegeben, als Kantone Impfungen von gefährdeten Bevölkerungsgruppen, von besonders exponierten Personen und 


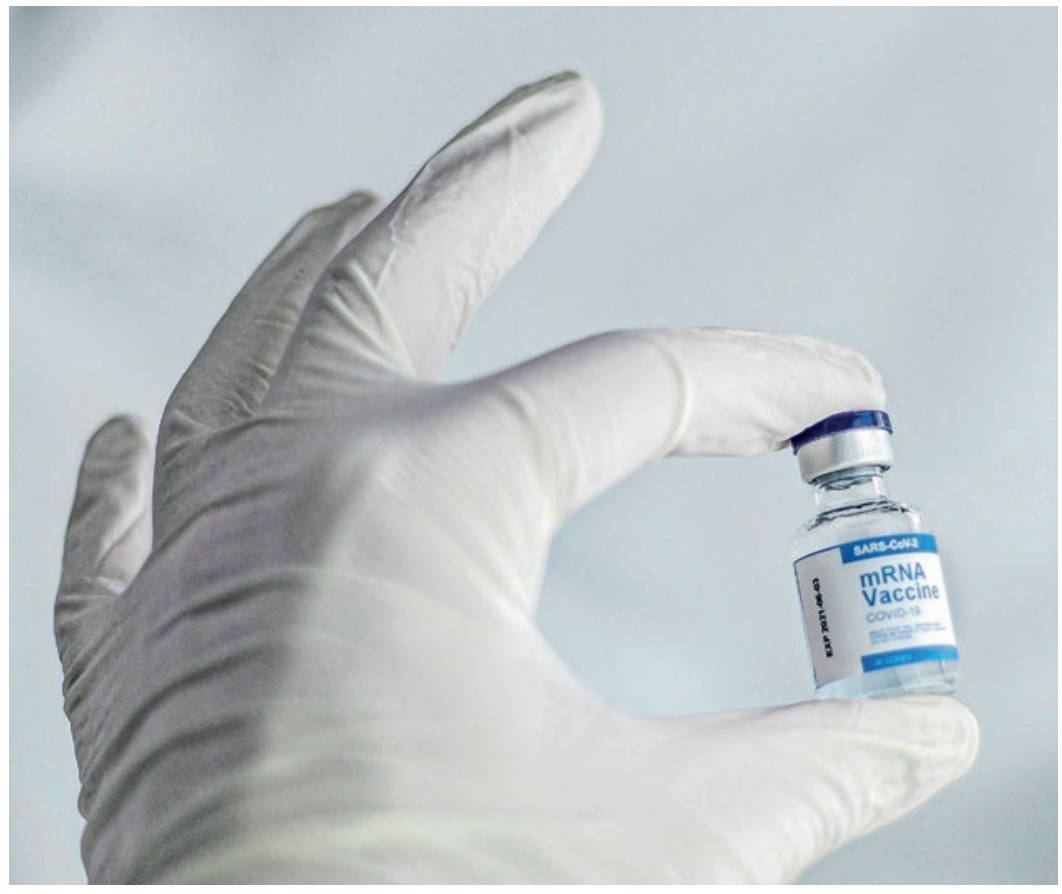

von Personen, die bestimmte Tätigkeiten ausüben, für obligatorisch erklären können, sofern eine erhebliche Gefahr besteht.

Anhand von Art. 38 «Obligatorische Impfungen» Epidemienverordnung ist die Prüfung durch die zuständigen kantonalen Behörden vorzunehmen, ob eine erhebliche Gefahr gemäss Art. 22 EpG besteht. Eine wichtige Rolle spielen dabei namentlich

- der Schweregrad einer möglichen Erkrankung sowie das Risiko einer Weiterverbreitung der Krankheit,

- die Gefährdung besonders vulnerabler Personen,

- die epidemiologische Situation auf kantonaler, nationaler und internationaler Ebene unter Einbezug des BAG,

- die zu erwartende Wirksamkeit eines allfälligen Impfobligatoriums und

- die Eignung und Wirksamkeit anderer Massnahmen zur Eindämmung der Gesundheitsgefahr.

In Art. 38 Abs. 2 Epidemienverordnung heisst es weiter: «Ein Impfobligatorium für Personen, die bestimmte Tätigkeiten ausüben, insbesondere in Gesundheitseinrichtungen, ist auf diejenigen Bereiche zu beschränken, in welchen das Risiko einer Weiterverbreitung der Krankheit erhöht ist oder in welchen besonders verletzbare Personen gefährdet sind.» In zeitlicher Hinsicht ist dieses Obligatorium zu befristen, des Weiteren darf eine Impfung nicht mittels physischen Zwangs erfolgen.

\section{Bundeskompetenz}

Eine wichtige Bestimmung ist der nachfolgend zitierte Art. 6 Abs. 2 lit. d Epidemiengesetz (EpG). Demnach kann der Bundesrat bei Vorliegen einer besonderen Lage nach Anhörung der Kantone für vulnerable Personengruppen, besonders exponierte Personen oder Personen, die bestimmte Tätigkeiten ausüben, ein Impfobligatorium vorsehen.

\section{Art. 6 Abs. 2 EpG}

2 Der Bundesrat kann nach Anhörung der Kantone folgende Massnahmen anordnen:

a. Massnahmen gegenüber einzelnen Personen;

b. Massnahmen gegenüber der Bevölkerung;

c. Ärztinnen, Ärzte und weitere Gesundheitsfachpersonen verpflichten, bei der Bekämpfung übertragbarer Krankheiten mitzuwirken;

d. Impfungen bei gefährdeten Bevölkerungsgruppen, bei besonders exponierten Personen und bei Personen, die bestimmte Tätigkeiten ausüben, für obligatorisch erklären.

Das Covid-19-Gesetz bietet für den Bundesrat hingegen keinerlei Grundlage, in der gegenwärtigen besonderen Lage direkt oder indirekt über Art. 6 Abs. 2 lit. d EpG hinausgehende Impfobligatorien anzuordnen [6].

Des Weiteren kann der Bundesrat gemäss Art. 7 Ausserordentliche Lage EpG für das ganze Land oder für einzelne Landesteile die notwendigen Massnahmen anordnen, wenn es eine ausserordentliche Lage erfordert. Dies ist dann relevant, wenn eine übertragbare Krankheit, die eine schwere Bedrohung der öffentlichen Gesundheit darstellt, plötzlich und unvorhersehbar auftritt.

\section{Verhältnismässigkeitsprinzip}

Einschränkungen von Grundrechten bedürfen einer gesetzlichen Grundlage [7]. Sie müssen durch ein öffentliches Interesse oder durch den Schutz von Grundrechten Dritter gerechtfertigt und verhältnismässig sein [8]. Ob öffentliche Interessen eine Grundrechtseinschränkung rechtfertigen, lässt sich im Zusammenhang mit einem Impfobligatorium nur basierend auf medizinisch-epidemiologischem Fachwissen beantworten. Eine gesetzliche Unbestimmtheit sei gemäss Bundesgericht durch das Verhältnismässigkeitsprinzip zu kompensieren. Demnach, so das Bundesgericht, kommt dem Verhältnismässigkeitsgrundsatz «besondere Bedeutung zu für die harmonisierende Konkretisierung konfligierender Verfassungsprinzipien, wie zum Beispiel dem Schutz von Leben und Gesundheit einerseits und den zu diesem Zweck verhängten Grundrechtseinschränkungen andererseits» [9]. Und die Verhältnismässigkeit 
müsse gemäss der Bundesrichter umso strenger geprüft werden, «wo die Unbestimmtheit von Rechtssätzen zu einem Verlust von Rechtssicherheit führt».

\section{Impfobligatorium und Impfzwang}

In der juristischen Lehre wird der Unterschied zwischen Impfobligatorium und Impfzwang beleuchtet [10]. Für ein Impfobligatorium bestehen bei besonderer und ausserordentlicher Lage unter dem Vorbehalt des Verhältnismässigkeitsprinzips Rechtsgrundlagen. Hingegen gibt es im Epidemiengesetz für einen Impfzwang keine gesetzliche Grundlage. Dies lässt sich aus Art. 32 EpG ableiten, welcher die Massnahmen abschliessend aufzählt, die zwangsweise durchgesetzt werden können.

\section{Das Impfobligatorium aus arbeits- rechtlicher Sicht [11]}

Ein Impfobligatorium des Arbeitgebers müsse gemäss den Autoren Sturny und Zendeli «durch die betriebliche Tätigkeit begründet sein und es darf keine milderen Schutzmassnahmen (zum Beispiel Anzüge, Masken etc.) geben, die zum selben Ergebnis führen würden» [12]. Laut Hug und Pärli fehlt für eine abschliessende Beurteilung sowohl eine Klärung als auch eine wissenschaftliche Analyse dieser Vertragseinschränkung im Hinblick auf eine privatrechtliche Impfpflicht im Arbeitsvertrag [13]. Unter dem Aspekt der Verhältnismässigkeit sei eine Abwägung zwischen Schutz des Patienten und dem Eingriff in die körperliche Unversehrtheit zum Beispiel des Gesundheitspersonals vorzunehmen.

Der Arbeitsvertrag würde somit grundsätzlich die Möglichkeit bieten, in privatrechtlichen Gesundheitsbetrieben ein Covid-19-Impfobligatorium mit den Arbeitnehmenden zu vereinbaren. Bei einem vertraglich vereinbarten Impfobligatorium würde eine Nichtimpfung eine Vertragsverletzung darstellen, was
Arbeitgebende mit Massnahmen bis einschliesslich der Kündigung sanktionieren könnten.

\section{Bildnachweis}

Ivan Diaz / Unsplash

Spencer Davis / Unsplash

\section{Literatur}

1 Die Impfpflicht ist menschenrechtskonform, NZZ Nr. 89, 19.4.2021, S. 19; Richter billigen Impfobligatorium in Tschechien (aerztezeitung.de); VAVŘIČKA AND OTHERS v. THE CZECH REPUBLIC (coe. int) (letzter Zugriff am 6.10.2021).

2 Urteil (des Bundesgerichts) 2C_941/2020 vom 8. Juli 2021 E. 3.2.3, zur Publikation vorgesehen.

3 Art. 2 Abs. 1 EpG.

4 Die Verordnung über die Bekämpfung übertragbarer Krankheiten des Menschen (Epidemienverordnung, EpV) regelt insbesondere die Überwachung, Verhütung und Bekämpfung von übertragbaren Krankheiten.

5 Botschaft zum Bundesgesetz über die gesetzlichen Grundlagen für Verordnungen des Bundesrates zur Bewältigung der Covid-19-Epidemie (Covid-19-Gesetz), BBl 20206563 6621; https://www.fedlex. admin.ch/eli/fga/2020/1669/de

6 «Die Möglichkeit, ein Impfobligatorium anzuordnen bei gefährdeten Bevölkerungsgruppen, bei besonders exponierten Personen und bei Personen, die bestimmte Tätigkeiten ausüben (z.B. Arbeit auf bestimmten Abteilungen in Spitälern oder in Heimen), ergibt sich aus dem geltenden Epidemiengesetz (Art. 6 Abs. 2 Bst. d EpG). Das vorgesehene Covid-19-Gesetz enthält keine diesbezügliche Grundlage. Ein Impfobligatorium bedeutet nicht das Gleiche wie ein Impfzwang. Weder besteht eine gesetzliche Grundlage, gestützt auf die jemand unter Zwang geimpft werden könnte, noch ist eine solche im Covid-19-Gesetz oder in einem anderen Erlass vorgesehen.» (Botschaft zum Bundesgesetz über die gesetzlichen Grundlagen für Verordnungen des Bundesrates zur Bewältigung der Covid-19-Epidemie [Covid-19-Gesetz]) BBl 202065636581 [https://www.fedlex.admin.ch/eli/fga/2020/1669/de]); Kaspar Gerber, Drei Leiturteile des Bundesgerichts zu kantonalen Coronamassnahmen, in: Jusletter 16. August 2021, N 96, mit weiteren Nachweisen.

7 Art. 36 Abs. 1 BV

8 Art. 36 Abs. 2 und 3 BV.

9 Urteil (des Bundesgerichts) 2C_941/2020 vom 8. Juli 2021 E. 3.2.3, zur Publikation vorgesehen.

10 Vokinger KN, Rohner N. Impfobligatorium und Impfzwang - eine staatsrechtliche Würdigung. recht 2020, S. 257ff.

11 Szucs TD, Drack SF. Covid-19-Impfobligatorium, Möglichkeiten und Haftung von privatrechtlichen Arbeitgebern von Gesundheitspersonal, in Jusletter 14. Juni 2021.

12 Sturny T, Zendeli D. Gesetzgebung und Rechtsprechung, RRVR 2/2021, S. 11.

13 Hug J, Pärli K. Impfzwang in Gesundheitsbetrieben des Privatrechts. Pflegerecht. 2013;3:165-73. 